\title{
EDITORIAL
}

Takayuki Itoh

\section{Visual information communication and interaction (VINCI 2015)}

Published online: 23 February 2017

(C) The Visualization Society of Japan 2017

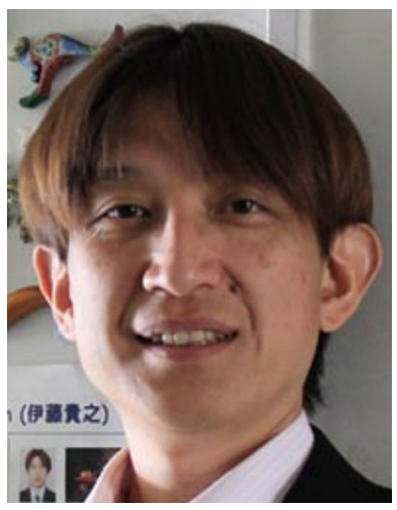

The 8th International Symposium on Visual Information Communication and Interaction (VINCI 2015) has been held in Tokyo, Japan, during 24-26 August, 2015. The state-of-the-arts in many aspects of visualization methodologies and applications, and visual design/art were presented and discussed. In total, 32 papers were submitted from 13 countries. After a rigorous peer review process where each paper was reviewed by three reviewers, 12 full papers and 8 short papers were selected for presentation. Prof. Kumiyo Nakakoji from Kyoto University, Japan, gave a keynote speech titled "Designing Visual Interactivity for Data Experience and Engagement." Prof. Raimondo Schettini from University of Milano-Bicocca, Italy, gave a keynote speech titled "Content-aware Color Constancy". In addition, VINICI 2015 featured a tutorial "Visually Do Statistics for Business Persons".

After the symposium, authors of the full papers were invited to submit extended versions to Journal of Visualization (JOV), and six papers were finally accepted for publication by the standard peer review process of JOV. We wish to thank the editors and reviewers of JOV for making it possible to publish this special feature from VINCI 2015. We also thank the authors for their careful and insightful work and cooperation in the preparation of the revised papers. It will be our pleasure if readers appreciate the hot topics in visualization research as a result of this special feature. We would like to express our sincere thanks to the staff at Springer-Verlag for their kind support.

T. Itoh $(\bowtie)$

Ochanomizu University, Tokyo, Japan

E-mail: itot@is.ocha.ac.jp 